MATEC Web of Conferences 51, 01006 (2016)

DOI: $10.1051 /$ matecconf $/ 20165101006$

C Owned by the authors, published by EDP Sciences, 2016

\title{
Methodology for Structural Calculation of Gear Teeth with Unconventional Profile
}

\author{
Andrea Chiaramonte Radicella ${ }^{1}$, Giulio Di Francesco ${ }^{2, a}$, Vincenzo La Battaglia ${ }^{2}$ and Stefano Marini ${ }^{2}$ \\ ${ }^{1}$ Toyota Motor Europe, Avenue Du Bourget 60, 1140 Ville de Bruxelles, Belgium \\ ${ }^{2}$ Università degli Studi Roma Tre - Dipartimento di Ingegneria, Via della Vasca Navale 79, 00146 Roma, Italy
}

\begin{abstract}
After having made reference to the structural analysis used in the study of gear wheel teeth, we then move on to the state of the art on the topic. We proceed to identify the boundary conditions used in the structural analysis of unconventional teeth with sides having a profile of an involute of a circle but with different pressure angles in each of the two sides. A procedure for the discretization of traditional teeth and of innovative teeth is presented and compared with the discretization obtained using current software.
\end{abstract}

\section{General details}

Gear wheels with teeth having sides with different pressure angles are explored further given their advantages, especially in some applications, over traditional teeth [1] [2]. With the center distance being the same, they are stronger which means that, for a given torque, smaller gears can be used [3] [4] [5].

Hence the importance of analyzing further the structural characteristics of said tooth and, in particular, of identifying a method for automatically subdividing the structure into elements beforehand [6] [7].

We now deal with the issue of the Finite Elements Method mesh of the structure of the tooth having two sides with different involutes.

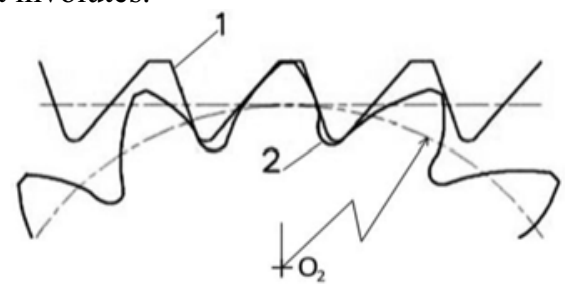

Figure 1. Asymmetric gear engaged.

The Figure 1 shows a gear wheel (2) with a tooth having pressure angles $\alpha_{01}$ and $\alpha_{02}$ which engages with an infinite radius wheel (1), also having the same corresponding angles $\alpha_{01}$ and $\alpha_{02}$. Contact conditions between active flanks (right for wheel 2 in Fig. 1) are the same we have in traditional teeth.

\footnotetext{
${ }^{a}$ Corresponding author : difrance@uniroma3.it
} 


\section{Current state of the art}

It has been pointed out that the use of advanced and current structure modelling software traditional teeth does not generally allow to obtain a mesh that can be used for subsequent in-depth FEM structural calculations.

These generic software applications fail to achieve the necessary accuracy for discretization and for the subsequent structural calculation stage. This drawback occurs in some areas in which a denser mesh would in fact be required; generally, the choice of a suitable tooth mesh density must take into account the stresses occurring during operation.

The meshing system that we refer to is based on the subdivision of the tooth through a network of "meridians" and "parallels": the "meridians" are roughly composed of circular arcs, the "parallels", on the other hand, are curves with a trend similar to that of the tooth profile.

\section{Delimiting the tooth area to be discretized}

Based on the foregoing considerations, it is therefore necessary to propose a type of discretization that can be used in the subsequent stage of structural analysis of the tooth; to that end, the area of the tooth to be discretized needs to be delimited. We then conduct a preliminary analysis of the entire structure (gear) using a non-dense subdivision and identifying beforehand the areas of the tooth under significant stresses; in this way, we can delimit the area of the tooth to be processed with a more dense discretization in order to achieve a more in-depth structural analysis [8] [9].

The area thus defined, delimited by its external profile, is subdivided into elements using meridians and parallels branching off, perpendicularly to the external boundary profile, from one side of the tooth and extending perpendicularly to the external boundary profile of the opposite side.

Said meridians and parallels subdivide the structure into quadrilateral elements with the four sides being as parallel as possible between them, two by two, and not too dissimilar from one another in size.
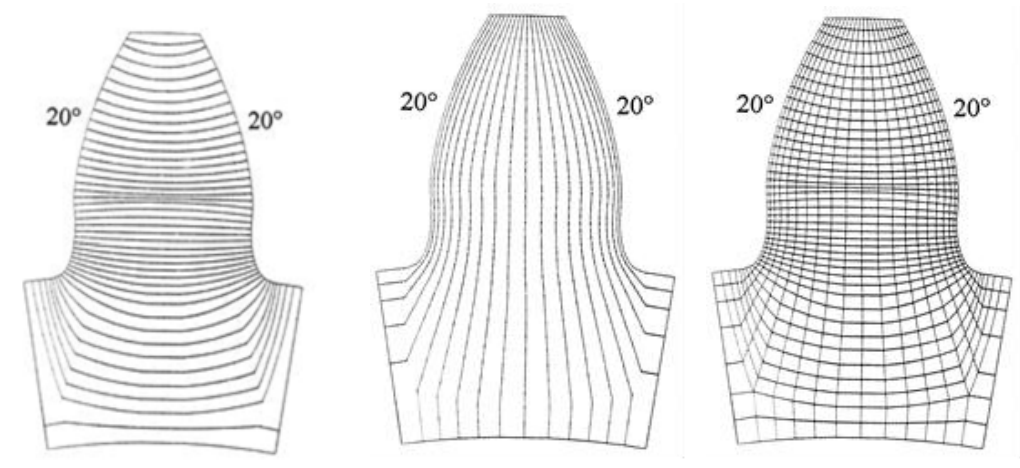

Figure 2. Parallels and meridians discretization

The lower part of the tooth model digs below the base root circle reaching a depth at which the stress is practically insignificant.

Fig. 2 shows a conceptual diagram of what is set out above.

In order to trace the tooth profile, we need to know its defining parameters including those of the tool that we plan to use for cutting the gear tooth.

\section{Discretization of traditional teeth}

Fig. 2 shows the discretization of a traditional tooth using the parallels and meridians referred to above and, lastly, the fully discretized tooth. 
As regards the discretization of the tooth structure, we noted that the use of advanced and current structure modelling software applications for traditional teeth does not generally allow (Fig.3, software Comsol 5.1) to obtain a mesh that can be used for the subsequent in-depth FEM structural analysis.

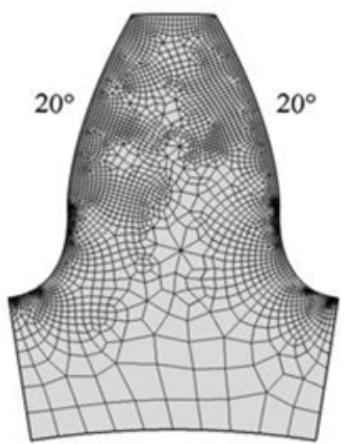

Figure 3. Mesh obtained using commercial software

As these figures clearly show, said generic applications fail to achieve the necessary precision for the discretization and the subsequent structural analysis stage; this drawback occurs especially in some areas in which a more dense mesh would in fact be required; generally, the choice of a suitable tooth mesh density must take into account the stresses occurring during operation.

As stated above, the discretization example obtained using current excellent modelling software applications (software Comsol 5.1) is not satisfactory in terms of the accuracy required in the structural analysis of this specific case of innovative teeth; the areas with a denser and more homogeneous mesh may indeed not fully correspond with those subject to higher tensile stresses.

\section{Discretization of innovative teeth}

The figure below shows a tooth characterized by $\alpha_{01}=14^{\circ}$ and $\alpha_{02}=20^{\circ}$ (Fig. 4 left), $\alpha_{01}=20^{\circ}$ and $\alpha_{02}=26^{\circ}$ (Fig. 4 center), obtained through the procedure proposed in this study and a corresponding tooth $\alpha_{01}=20^{\circ}$ and $\alpha_{02}=26^{\circ}$ (Fig. 4 right), obtained using the current commercial software.

As the images clearly show, the drawbacks referred to above are all the more evident when we move from the traditional structure $\left(20^{\circ}-20^{\circ}\right)$ to the innovative one $\left(20^{\circ}-26^{\circ}\right)$, object of this indepth study.

As stated above, meridians and parallels subdivide the structure into four-sided elements with the four sides being as parallel as possible between them and not too dissimilar from one another in size.

The area to be analyzed more in depth is obtained by joining the nodes whose shift is greater than a value that is considered non-significant.
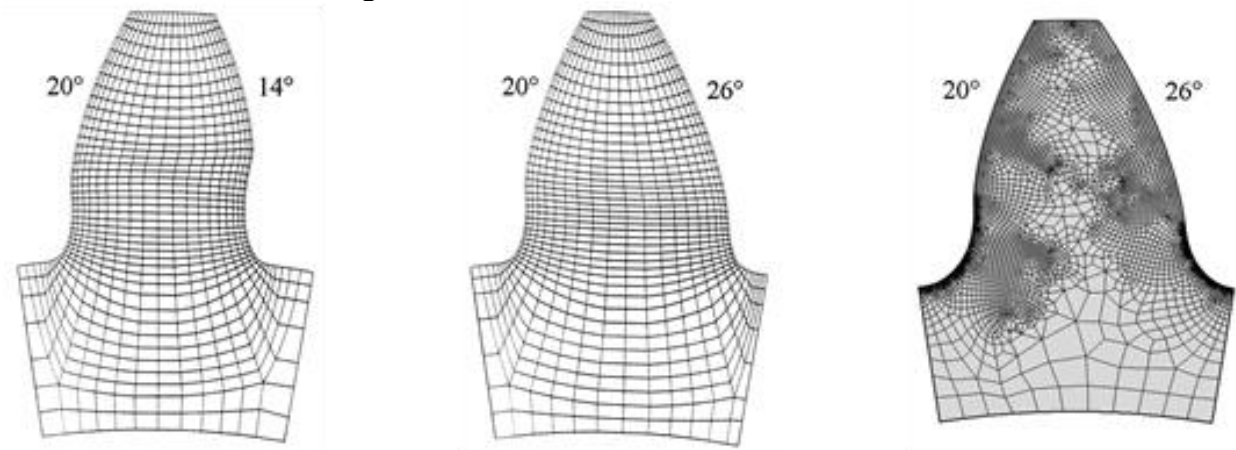

Figure 4. Different meshes for asymmetrical teeth. 


\section{MATEC Web of Conferences}

The structure of the lower part of the tooth protrudes below the root circle up to a depth at which the stresses are practically irrelevant.

The software under analysis must first allow to obtain an automatic discretization of the structure; once the innovative tooth, using the traditional method, has been identified from a geometrical perspective, (base circle, addendum circle, left involute with pressure angle $\alpha_{01}$, right involute with pressure angle $\alpha_{02}$, trochoidal profile fillet, in the case of rounded edge tools, for one side and the other side of the tooth, etc.) we then proceed, based on the multitude of parameters obtained through the calculations, to the automatic discretization of the structure and to the subsequent structural analysis.

The result obtained is shown in the figures above; as you can see, we obtain an appropriate density of elements which gradually move from the outside inwards, compatibly with the stresses arising in the structure when engaging with the tooth of the companion gear. Noteworthy is the orderly increase in the density of the elements in the fillet areas and their obvious branching off at the base of the tooth structure under analysis (non significant stresses).
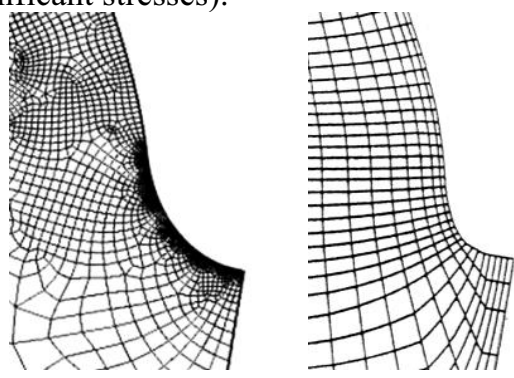

Figure 5. Increase in the density of the elements obtained with Commercial Software (left) and with the software under analysis

The increase of density of the elements obtained with the software under analysis produces elements having similar geometry of the other elements but with smaller dimension. This effect produces regular elements in the whole mesh. The commercial software produces a mesh with irregular elements characterized by different form and geometry. Figure 5 shows the described difference.

In this particular case, as is evident, the "parallels" cannot simply be arcs of a circle, but rather complex curves which are identified, defined and described automatically from time to time.

Similarly to the case of traditional gear discretization, also in this case we identify a certain number of points gradually separated from each other; a meridian is drawn through each point.

Each meridian consists of a curve passing through all points of the same general index placed on each of the parallels examined above.

In reality, it is no easy task to provide a generalized modelling system for this type of toothing.

Thanks to the system adopted, it is therefore possible to select the areas in which to automatically increase the density of discretization as a function of the stress fields during engagement; said fields may vary depending on the application of the gears.

In the case of gears for gear pumps, it is necessary to know the position of the force transferred between the two engaging teeth and to bear in mind that below or above the contact point between the teeth the stress field is represented by a hydrostatic pressure which is high on one side and equal to the suction pressure on the other side; hence, in such case the stress field is more complex and must be handled with great precision

\section{Conclusions}

These innovative wheels (different pressure angles for the two sides of the tooth: $\alpha_{01}$ and $\alpha_{02}$ ) have some considerable advantages. The tooth is more robust: with other factors being equal, they can transmit a higher torque; on equal torque to be transmitted, their overall size is reduced. 
The geometrical difference varies as the angles $\alpha_{01}$ and $\alpha_{02}$ of the two sides of the tooth, the left side and the right side, vary [10]. The variation in the second pressure angle, permits to vary the robustness of the tooth and hence to increase transmission performance [11]. However, the quantification of this increase in performance cannot be estimated other than through a prior FEM analysis of the tooth [12]. This analysis is in any case limited by the absence of an automatic and efficient mesh generator for the tooth structure.

The procedure adopted allows to conduct the structural analysis of traditional teeth and of innovative teeth accurately and rapidly: the use of advanced analysis software is made easier by starting with an accurate subdivision into elements.

The procedure under analysis is of a general nature and allows to obtain reliable results with short calculation times [13].

The mathematical model is complex and that depends in particular on the need to have a general tool that can be used for the various applications for which the gears of a given assembly are intended: transmission gears, gears for gear pumps, etc.

\section{References}

1. A. Sanders, D. R. Houser, A. Kahraman, J. Harianto, S. Shon, “An experimental investigation of the effect of tooth asymmetry and tooth root shape on root stresses and single tooth bending fatigue life of gear teeth." Proceedings of the ASME Design Engineering Technical Conference, 8, pp. 297-305. (2011).

2. R. Thirumurugan, C.C.C. Deepak, "Design and analysis of asymmetrical spur gear drive for automobile gear box application", Applied Mechanics and Materials, 592-594, pp. 2277-2281, (2014).

3. A. Kapelevich, "Direct gear design for asymmetric tooth gears" Mechanisms and Machine Science, 34, pp. 117-143, (2016)

4. S. Ekwaro-Osire, I. Durukan, F.M. Alemayehu, "Experimental and probabilistic analysis of asymmetric gear tooth. Conference Proceedings of the Society for Experimental Mechanics Series, 5, pp. 207-212, (2011)

5. G. Di Francesco, S. Marini, "Structural Analysis of Asymmetrical Teeth: Reduction of Size and Weight" Gear Technology, Randall Publishing inc. Sept- Oct. 1997, Elk Grove Village IL. U.S.A.(1997)

6. G. Di Francesco, S. Marini, "Asymmetric teeth: bending stress calculation", Gear Technology (Mar-Apr 2007), Randall Publishing inc., Elk Grove Village, IL USA (2007)

7. G. Di Francesco, S. Marini, "Tensioni di flessione di dentature asimmetriche e dimensionamento", Organi di Trasmissione (April 2005), Tecniche Nuove, Milan (2005)

8. G. Di Francesco, S. Marini, "Asymmetrical gear wheels: automatized procedure for the design", International Conference on Gears, Munich 2005

9. G. Di Francesco, S. Marini, "Ruote a denti asimmetrici: procedura automatizzata per il dimensionamento", Organi di Trasmissione (February 2008), Tecniche Nuove, Milan (2008)

10. G. Di Francesco, S. Marini, G. Grasso, C. Clienti, "Asymmetric gear wheel thermographic analysis", Colloque Photomecanique, Étude du comportement des matèriaux et des structures, Albi (2004)

11. G. Di Francesco, S. Marini, “Asymmetrical gear wheel design: determining the bending stress in relation to the degree of asymmetry", TMT 2004, Trends in the development of machinery and associated technology, Neum (2004)

12. G. Di Francesco, S. Marini, "Dentature asimmetriche: evoluzione e procedura analitica per il dimensionamento" Organi di Trasmissione, Tecniche Nuove, Milano, (1998)

13. G. Di Francesco, S. Marini, "Adattamento della procedura ISO C alle dentature asimmetriche", XXXIV Convegno AIAS, Milan (2005) 